\title{
Qualidade da água, capacidade de suporte e melhor período para criação de peixes em tanques-rede no reservatório de Salto Caxias
}

\author{
Water quality, capacity of holder and best time \\ to fish farming in cages in the reservoir Salto Caxias
}

Irineu Frederico Feiden', José Dilson Silva de Oliveira², Odair Diemer ${ }^{3}$, Aldi Feiden ${ }^{4}$

口-

\begin{abstract}
RESUMO
O controle das variáveis abióticas na criação de peixes em tanques-rede é difícil devido às estruturas serem distribuídas ao longo de áreas extensas, assim, o conhecimento do nível máximo de produção suportável pelo ambiente é de suma importância para que a atividade piscícola ocorra de forma sustentável e ambientalmente correta. O estudo teve como objetivo avaliar as características limnológicas durante o período de um ano na área de abrangência da criação de peixes em tanques-rede no reservatório de Salto Caxias, Rio Iguaçu, e com os resultados obtidos determinar a capacidade de suporte do local, além de identificar a melhor época de produção de tilápias em função da temperatura da água. As variáveis avaliadas foram: temperatura da água, potencial hidrogeniônico, oxigênio dissolvido, transparência da água, clorofila, fósforo total e ortofosfato. De modo geral, o local apresenta características propícias para criação de peixes, não havendo indícios de deterioração da qualidade de água. Entre os meses de dezembro e março as temperaturas da água foram mais favoráveis para o cultivo. A capacidade de produção do local pode ser aumentada em 663 toneladas sem exceder o limite de fósforo total recomendado na resolução 357/05 do CONAMA.
\end{abstract}

Palavras-chave: aquicultura; limnologia; sustentabilidade; cultivo superintensivo.

\begin{abstract}
The control of abiotic variables on fish farming in cages is difficult because the structures are distributed over large areas, so, the knowledge of the maximum level of production supportable by the environment is of great importance, so that the activity can occur in a sustainable way and being environmentally correct. The study aimed to evaluate the limnological characteristics during the period of one year in the area of fish farming in cages in Salto Caxias Reservoir, Iguaçu River; and with the results, to determine the capacity that the local can support, and to identify the best Oreochromis niloticus production period, depending on water temperature. The variables evaluated were: water temperature, hydrogen potential, dissolved ox ygen, water clarity, chlorophyll, total phosphorus and orthophosphate. In general, the local has characteristics conducive to raising fish, with no evidence of deterioration of water quality. Between the months of December and March, water temperatures were more favorable to the creation. The production capacity of the local can be increased in 663 tons, without exceeding the recommended limit of phosphorus in resolution 357/05 of CONAMA.
\end{abstract}

Keywords: aquaculture; limnology; sustainability; superintensive creation.

\section{INTRODUÇÃO}

A pesca e a aquicultura são consideradas pela Organização das Nações Unidas (ONU) como atividades estratégicas para a segurança alimentar sustentável do planeta, pois são capazes de fornecer alimento proteico de alta qualidade e de gerar emprego tanto em países desenvolvidos como em desenvolvimento (ARANA, 2007). Nesse sentido, Tundisi (2005) menciona que os reservatórios artificiais têm sido utilizados para múltiplas finalidades, entre elas a produção de alimento por meio da piscicultura. Assim, a gestão e o monitoramento da qualidade hídrica dos reservatórios se tornam fundamentais para os usos múltiplos (ECHANIZ \& VIGNATTI, 2009).

O Rio Iguaçu, no estado do Paraná, possui diversos reservatórios de hidroelétricas, como Salto Caxias, Segredo, Salto Santiago e Salto Osório, instalados em seu leito para a geração de energia elétrica. Recentemente tem despertado o interesse para a produção de peixes em tanques-rede (IAP, 2012). Para o fomento dessa atividade na bacia

'Mestre em Recursos Pesqueiros e Engenharia de Pesca pela Universidade Estadual do Oeste do Paraná (UNIOESTE) - Toledo (PR), Brasil. 2Doutor em Química Analítica. Professor da UNIOESTE - Toledo (PR), Brasil.

3Doutor em Aquicultura. Professor do Instituto de Educação, Ciência e Tecnologia do Mato Grosso do Sul (IFMS) - Coxim (MS), Brasil.

${ }^{4}$ Doutor em Ecologia de Ambientes Aquáticos Continentais. Professor da UNIOESTE - Toledo (PR), Brasil.

Endereço para correspondência: Odair Diemer - Rua Travessa Olinda, 35 - Morada Alto São Pedro - 79400-O0 - Coxim (MS), Brasil - E-mail: odairdiemer@hotmail.com Recebido: 03/04/13 - Aceito: 28/01/15 - Reg. ABES: 114845 
do Iguaçu, é indispensável obter informações referentes às características limnológicas dos locais de produção, para, sobretudo, averiguar a viabilidade.

Um dos principais fatores que podem inviabilizar a criação de tilápias na região é o clima. O clima na Unidade Hidrográfica do Iguaçu é subtropical com temperatura média no mês mais frio inferior a $18^{\circ} \mathrm{C}$ e temperatura média no mês mais quente acima de $22^{\circ} \mathrm{C}$, com verões quentes, geadas pouco frequentes e tendência de concentração de chuvas nos meses de verão (CABI, 2012). Segundo Kubitza (2000), a temperatura da água ideal para as tilápias (Oreochromis niloticus) está na faixa de 27 a $32^{\circ} \mathrm{C}$.

Outro aspecto a ser considerado é o fato da piscicultura ser uma atividade potencialmente poluidora, o que demanda preocupações. Todavia, resultados indicam que esse cultivo pode ser praticado com sustentabilidade quando alguns cuidados são adotados, especialmente respeitando-se os limites da capacidade de suporte (DIEMER et al., 2010). O controle dos parâmetros abióticos na criação de peixes em tanques-rede é difícil devido às estruturas serem distribuídas ao longo de áreas extensas. Desse modo, o conhecimento do nível máximo de produção suportável pelo ambiente é de suma importância para que a atividade piscícola ocorra de forma sustentável e ambientalmente correta (LIU et al., 2008).

Assim, o presente estudo teve como objetivo fazer um monitoramento das características limnológicas durante o período de um ano na área de abrangência da criação de peixes em tanques-rede no reservatório de Salto Caxias, Rio Iguaçu, verificando se ocorrem variações temporais na qualidade da água e com os resultados obtidos determinar a capacidade de suporte do local, além de identificar a melhor época de produção de tilápias em função da temperatura da água.

\section{METODOLOGIA}

As coletas foram conduzidas na área de criação de peixes em tanques-rede, localizada no Centro de Desenvolvimento e Difusão de Tecnologia (CDT-Iguaçu), município de Boa Vista da Aparecida, Paraná, reservatório da Usina Hidroelétrica Governador José Richa (Salto Caxias) no Rio Iguaçu, onde são criadas tilápias (Oreochromis niloticus) e espécies endêmicas do Rio Iguaçu, como jundiá (Rhamdia voulezi), mandi-pintado (Pimelodus britskii) e surubim do Iguaçu (Steindachneridion melanodermatum).

O estudo foi realizado durante os meses de agosto de 2010 a julho de 2011, com coletas mensais. Para o levantamento dos dados abióticos, estabeleceram-se seis estações de amostragem, de modo que toda a área dos tanques-rede fosse abrangida. Cada local foi considerado como uma repetição. As estações foram assim distribuídas: P1, localizada a montante do rio Jacutinga; P2, local de coleta dentro do perímetro dos tanques-rede; P3, local 100 metros acima dos tanques-rede;
P4, local em frente ao deposito de rações; P5, local a uma distância de 500 metros abaixo dos tanques-rede; e P6, confluência do Remanso e do Rio Jacutinga (Figura 1).

Os parâmetros avaliados foram: temperatura da água, potencial hidrogeniônico $(\mathrm{pH})$, oxigênio dissolvido, mensurados in loco com emprego de um potenciômetro portátil da marca Hanna ${ }^{\oplus}$ Instruments com leitura digital, transparência da água obtida por meio do desaparecimento visual do disco de Secchi; clorofila analisada segundo metodologia descrita por Sipaúba-Tavares (1995); fósforo total e ortofosfato de acordo com APHA (1998).

Para análise da temperatura da água, as amostras foram coletadas com auxilio de uma garrafa de Van Dorn em três estratos: epilímnio (camada superficial), determinado por $0,54 \%$ da transparência da água; metalímnio (camada intermediária), verificado através de 1,7\% da transparência; e hipolímnio (camada profunda), sendo a profundidade máxima do local, conforme Wetzel (1993).

Para análise de clorofila, fósforo total e ortofosfato, foram coletadas amostras de água, preservadas em garrafas de polietileno escuras e conservadas resfriadas para posterior análise em laboratório.

A estimativa da capacidade de suporte para a criação de tilápias na área aquicola do CDT-Iguaçu foi efetuada por meio do método desenvolvido por Dillon e Rigler (1974). O modelo considerou a concentração média anual de fósforo total observado ao longo do período de estudo, ou seja, valores de fósforo total com o empreendimento em funcionamento.

O coeficiente de sedimentação do fósforo $(\mathrm{R})$ foi determinado segundo a equação: $R=1 /\left(1+0,614^{\star} \varphi^{\star} 0,491\right)$, em que: $\varphi=$ taxa de renovação da água do ambiente em número de vezes por ano, calculada como descrito por Santos (2001).

Para o cálculo da capacidade de suporte (L), foi utilizada a expressão: $\mathrm{L}=\mathrm{P}^{\star}\left(\mathrm{Z}^{\star} \varphi\right) /(1-\mathrm{R})$, onde: $\mathrm{P}=$ limite máximo para lançamento de fósforo total para o ambiente obtido da fórmula: $\mathrm{P}=\mathrm{PF}-\mathrm{PI}$, onde: $\mathrm{PI}=$ concentração de fósforo total médio mensurado durante o período de estudo e $\mathrm{PF}=$ concentração de fósforo total estabelecido na Resolução no 357/05, do CONAMA (2005); e Z=profundidade média do local.

Uma vez calculada a capacidade de suporte do local, foi estimada a quantidade máxima de tilápias que poderá ser produzida na área. Para tanto, foi considerada uma carga de $18,2 \mathrm{~kg}$ de fósforo lançado na água por tonelada de tilápia produzida e um período de cultivo de 180 dias e 2 ciclos de produção por ano, de acordo com a metodologia descrita por Kubitza (1999).

Os dados obtidos foram submetidos à análise de variância (ANOVA) e quando observadas diferenças significativas $(\mathrm{p}<0,05)$ foi aplicado o teste de Tukey a 5\% de significância (ZAR, 2009). Testes de normalidade e homocedasticidade das variâncias foram realizados, sendo as análises estatísticas efetuadas empregando-se o Software livre R-3.02. 


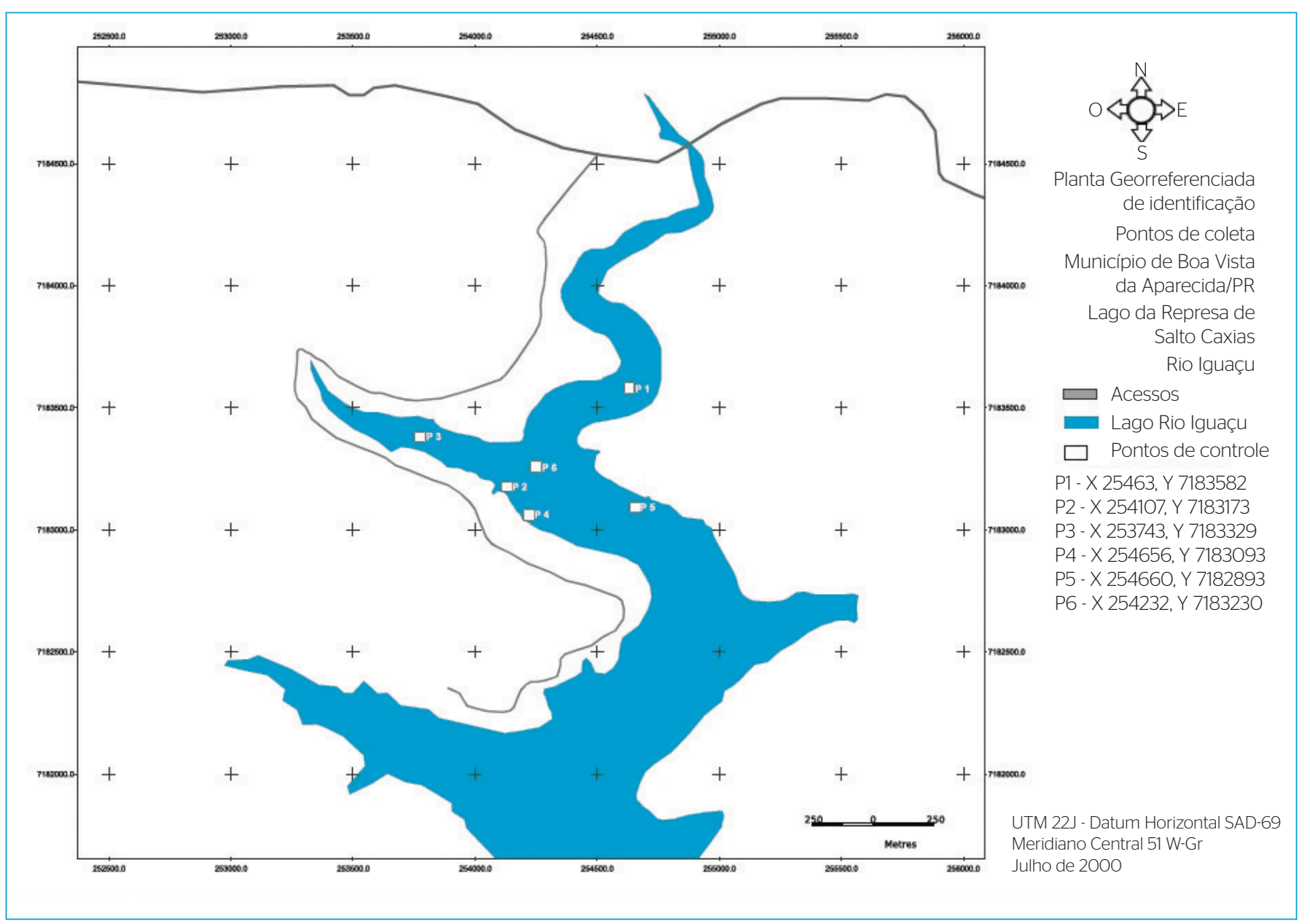

Figura 1 - Estações de amostragem na área de abrangência do Centro de Desenvolvimento e Difusão de Tecnologia Iguaçu.

\section{RESULTADOS E DISCUSSÃO}

As variáveis $\mathrm{pH}$ e clorofila não apresentaram diferença significativa entre os meses de amostrados ( $\mathrm{p}<0,05)$, conforme Tabela 1 . Entretanto, os parâmetros oxigênio dissolvido, transparência, fósforo total e ortofosfato diferiram estatisticamente ( $\mathrm{p}>0,05)$.

Houve variações entre os meses $(\mathrm{p}<0,05)$, menos para $\mathrm{pH}$ e clorofila, sendo que as concentrações de oxigênio foram maiores nos meses de junho e agosto com médias superiores a 9,0 mg.L-1 . Os teores desse elemento na água variam com a temperatura, bem como dependem ainda da pressão atmosférica (BOYD \& TUCKER, 1998). Em lagos, como foi o caso deste estudo, ocorre variação contínua do oxigênio, em consequência de processos físicos, químicos e biológicos (ESTEVES, 1998). No entanto, não foram verificados teores críticos para a criação de peixes.

Pereira e Bleich (2009), quando estudaram as variações limnológicas abióticas espaciais e temporais em uma lagoa na Bacia Araguaia-Tocantins, registraram concentrações de oxigênio dissolvido semelhantes e com oscilações de 2,7 a 8,6 mg.L $\mathrm{L}^{-1}$, e no presente estudo as variações foram de 5,79 a 12,06 mg. $\mathrm{L}^{-1}$.
A maior transparência foi averiguada no mês de setembro (4,39 m), enquanto as menores incidiram sobre os meses de novembro (1,29 m) e junho $(1,95 \mathrm{~m})$. As diferentes características de transparência da água podem estar associadas à pluviosidade e ao uso do solo nas áreas de captação do braço do reservatório, agravadas pela falta de matas ciliares nas encostas do lago. Conforme destaca Cabral et al. (2012), a presença principalmente de áreas de pecuária e plantio facilita a desagregação do solo pela ação das chuvas e o transporte de sólidos para os reservatórios.

De maneira geral, os valores das transparências foram superiores a dois metros. Segundo Schmittou (1993), ambientes aquáticos que apresentam transparência da água acima de dois metros de visualização pelo disco de Secchi proporcionam pouco enriquecimento em nutrientes, podendo ser utilizados para o cultivo de peixes em tanques-rede.

Os valores de fósforo total e ortofosfato apresentaram variações entre os meses de agosto de 2010 a julho de 2011. Apesar dessas oscilações, os níveis estavam dentro dos limites da Resolução n 357/05 do CONAMA (2005). Essas alterações podem ser atribuídas à movimentação das águas e pela liberação do fósforo do sedimento. Além disso, segundo Bueno et al. (2008), o cultivo de peixes pode influenciar na 
Tabela 1 - Valores médios de pH, oxigênio dissolvido, transparência, clorofila, fósforo total e ortofosfato nos diferentes meses de coleta na área de abrangência da criação de peixes em tanques-rede.

\begin{tabular}{|c|c|c|c|c|c|c|}
\hline \multirow[b]{2}{*}{ Mês/ano } & \multicolumn{6}{|c|}{ Variáveis } \\
\hline & $\mathrm{pH}$ & Oxigênio (mg. $\left.\mathrm{L}^{-1}\right)$ & Transparência (m) & Clorofila (mg. $\mathrm{L}^{-1}$ ) & $\begin{array}{l}\text { Fósforo total } \\
\left(\mathrm{mg} \cdot \mathrm{L}^{-1}\right)\end{array}$ & Ortofosfato (mg. $\left.\mathrm{L}^{-1}\right)$ \\
\hline Agosto/2010 & 7,14 & $10,53^{c}$ & $2,60^{\mathrm{bc}}$ & 0,0035 & $0,027^{h}$ & $0,006^{\mathrm{abc}}$ \\
\hline Setembro/2010 & 7,12 & $6,48^{a b}$ & $4,39 f$ & 0,0018 & $0,02 \mathrm{O}^{\mathrm{dfg}}$ & $0,006^{a b c}$ \\
\hline Outubro/2010 & 7,16 & $6,42^{\mathrm{ab}}$ & $3,14^{\text {cde }}$ & 0,0018 & $0,024^{\mathrm{fgh}}$ & $0,008^{c d}$ \\
\hline Novembro/2010 & 7,21 & $6,64^{\mathrm{ab}}$ & $1,29 a$ & 0,0036 & $0,025^{\mathrm{gh}}$ & $0,008^{c d}$ \\
\hline Dezembro/2010 & 7,40 & $6,60^{a b}$ & $3,27^{\text {cde }}$ & 0,0036 & 0,018 de & $0,005^{a b c}$ \\
\hline Janeiro/2011 & 7,13 & $7,34^{b}$ & $2,64^{\mathrm{bcd}}$ & 0,0025 & $0,029^{h}$ & $0,001^{a}$ \\
\hline Fevereiro/2011 & 7,35 & $6,35^{\mathrm{ab}}$ & $3,64^{e}$ & 0,0018 & $0,028^{h}$ & $0,002^{\mathrm{ab}}$ \\
\hline Março/2011 & 7,11 & $5,93^{a}$ & $3,33^{\text {de }}$ & 0,0020 & $0,017^{\mathrm{cd}}$ & $0,002^{a b}$ \\
\hline Abril/2011 & 7,13 & $5,79^{a}$ & 3,09cde & 0,0018 & $0,002^{a}$ & $0,002^{\mathrm{ab}}$ \\
\hline Maio/2011 & 7,29 & $7,24^{a}$ & 2,97cde & 0,0028 & 0,019def & $0,003^{a b c}$ \\
\hline Junho/2011 & 7,40 & $9,86^{c}$ & $1,95^{\mathrm{ab}}$ & 0,0010 & $0,011^{\mathrm{abc}}$ & $0,013^{d}$ \\
\hline Julho/2011 & 7,11 & $12,06^{c}$ & $2,86^{\mathrm{cd}}$ & 0,0023 & $0,005^{\mathrm{ab}}$ & $0,008^{c d}$ \\
\hline Coeficiente de variação (\%) & 7,21 & 7,99 & 12,42 & 59,98 & 18,63 & 51,75 \\
\hline
\end{tabular}

Valores na mesma linha seguidos de letra diferente diferem estatisticamente $(p>0,05)$ pelo teste de Tukey.

bioacumulação de fósforo no sedimento. Contudo, Freitas, Santos Silva e Darwich (2011), analisando a influência do cultivo de peixes em tanques-rede nos teores de nitrogênio e fósforo no lago Tupé em Manaus, Amazonas, relataram que a atividade não estava causando influências detectáveis.

Para o $\mathrm{pH}$ não foram detectadas diferenças significativas $(\mathrm{p}<0,05)$, sendo que o $\mathrm{pH}$ da água para peixes deve estar dentro do limite de 6,0 a 9,0 (BOYD \& TUCKER, 1998). Assim, as médias de pH estavam dentro do estabelecido para a criação de peixes, não verificando desequilíbrio para essa variável.

A clorofila expressa a biomassa fitoplânctonica e foi constante no local e apresentou concentrações baixas quando comparados aos dados encontrados por Eler et al. (2009), em viveiros de piscicultura, porém os sistemas são diferentes e com dinâmicas distintas.

Segundo Pereira e Mercante (2005), a assimilação de compostos nitrogenados pelo fitoplâncton pode acarretar crescimento descontrolado dessa comunidade, provocando florações de algas no ambiente. Como o local onde estão distribuídos os tanques-rede tem uma grande coluna de água, e pelas análises de clorofila apresentarem resultados amenos durante o ciclo anual, o que reforça a idéia de um local confortável para a criação de peixes.

As temperaturas da água ao longo dos 12 meses variaram significativamente $(\mathrm{p}<0,05)$. Nos meses de agosto e setembro de 2010 e junho de 2011, as temperaturas registradas foram mais baixas, o que prejudica a criação de tilápias, pois a zona confortável varia de 27 a $32^{\circ} \mathrm{C}$ (KUBITZA, 2000). Já nos meses de dezembro de 2010 a março de 2011, as temperaturas registradas foram mais elevadas, sendo favorável ao cultivo (Tabela 2).

A variação da temperatura da água no local está associada às estações do ano, que no sul do Brasil são bem definidas, oscilando entre primavera, outono, verão e inverno. A estratificação térmica no período de estudo foi evidente, tendo as temperaturas médias da água diminuído nas maiores profundidades, com exceção dos meses de novembro de 2010 e maio de 2011, que provavelmente estão associadas à dinâmica interna do reservatório, destacando o efeito da turbulência, induzido pelo vento superficial e pela diferença de densidade da água (ESTEVES, 1998).

Segundo Wetzel (1993), é conhecido que lagos e reservatórios apresentam um gradiente de temperatura da água de acordo com a profundidade, em geral associado às condições sazonais e climáticas, sendo que o fato esperado é que a amplitude térmica da água diminua com o aumento da profundidade, resultados visualizados no presente estudo.

Corroborando, Diemer et al. (2010), quando avaliaram a dinâmica nictimeral e vertical das características limnológicas em ambiente de criação de peixes em tanques-rede no município de Santa Helena, Paraná, reservatório da Itaipu Binacional, relataram similar comportamento térmico entre as diferentes profundidades.

A produção de tilápias no local pode ser aumentada, sem que seja ultrapassado o limite máximo de fósforo total recomendado na legislação (Tabela 3). No entanto, é importante destacar que na área já ocorre a criação (aproximadamente 10 toneladas anuais) e o valor obtido seria a capacidade de incremento da produção. 
Tabela 2 - Valores da temperatura da água em diferentes estratos na área de abrangência da criação de peixes em tanques-rede.

\begin{tabular}{|c|c|c|c|c|}
\hline Mês/ano & Epilimnio & Metalímnio & Hipolímnio & Coeficiente de variação (\%) \\
\hline Agosto/2010 & $20,35^{\mathrm{a}}$ & $18,61^{b}$ & $18,95^{b}$ & 3,42 \\
\hline Setembro/2010 & $20,58^{a}$ & $18,33^{\mathrm{ab}}$ & $18,60^{b}$ & 2,87 \\
\hline Novembro/2010 & $23,78^{a}$ & $22,55^{a}$ & $22,68^{a}$ & 4,82 \\
\hline Dezembro/2010 & $26,78^{a}$ & $24,53^{b}$ & $24,20^{b}$ & 4,01 \\
\hline Fevereiro/2011 & $27,68^{a}$ & $26,65^{a b}$ & $26,25^{b}$ & 2,64 \\
\hline Março/2O11 & $28,13^{a}$ & $24,91^{b}$ & $25,25^{b}$ & 1,64 \\
\hline Abril/2O11 & $25,51^{\mathrm{a}}$ & $24,53^{b}$ & $24,58^{b}$ & 1,45 \\
\hline Maio/2011 & $24,26^{a}$ & $24,10^{a}$ & $24,05^{a}$ & 1,24 \\
\hline Junho/2011 & $20,68^{a}$ & $19,70^{a}$ & $19,36^{b}$ & 4,51 \\
\hline
\end{tabular}

Valores na mesma linha seguidos da mesma letra não diferem estatisticamente $(p>0,05)$ pelo teste de Tukey.

Tabela 3 - Resultados do cálculo da capacidade de suporte da área aquícola do Centro de Desenvolvimento e Difusão de Tecnologia Iguaçu, localizado no município de Boa Vista da Aparecida, Paraná.

\begin{tabular}{l|c} 
Parâmetros & Valores obtidos \\
R - coeficiente de sedimentação & 0,30 \\
\hline P - limite máximo para lançamento de fósforo total & $0,011 \mathrm{mg} \cdot \mathrm{L}^{-1}$ \\
\hline Area calculada do braço do reservatório & $5.025 .747 \mathrm{~m}^{2}$ \\
\hline Profundidade média & $12 \mathrm{~m}$ \\
\hline L - capacidade de suporte & $13.266 \mathrm{~kg} \cdot \mathrm{P}^{-1} \cdot \mathrm{ano}^{-1}$ \\
\hline Produção estimada & 663,3 toneladas \\
\hline
\end{tabular}

No lago da Itaipu Binacional, Rio Paraná, a capacidade de suporte foi estimada em 6.237 toneladas de peixes por ano e instalação de 7.779 tanques-rede, nos três parques aquícolas demarcados e licenciados, com apoio da Itaipu Binacional. No entanto, estima-se que o potencial produtivo seja maior (INFORMATIVO CULTIVANDO ÁGUA BOA, 2009).

No reservatório de Sobradinho, Rio São Francisco, o dimensionamento da capacidade foi estimada em uma produção anual entre 4.687 e 12.503 toneladas de tilápia do Nilo, enquanto a estimativa da capacidade de suporte de todo o reservatório foi de 163.154 toneladas por ano (COSTA, 2004).
Em relação ao Rio Iguaçu, ainda não foram realizados estudos sobre a capacidade de produção de peixes em tanques-rede em seu leito. Contudo, como verificado neste estudo, o rio apresenta características favoráveis para esse sistema de criação, sendo uma importante alternativa para a diminuição da pressão sobre os recursos pesqueiros, além de gerar empregos e renda para as populações ribeirinhas. Esse sistema de produção é uma alternativa de investimento de menor custo e maior rapidez de implantação, sendo um agronegócio capaz de melhorar as condições sociais, ambientais e econômicas de uma região.

\section{CONCLUSÃO}

Mesmo sendo observadas variações nas características limnológicas, pode-se sugerir que o CDT-Iguaçu, reservatório de Salto Caxias, apresenta características propícias para a criação de peixes. Nos meses de dezembro de 2010 a março de 2011, as temperaturas da água foram mais favoráveis para a criação de tilápias e a capacidade de produção do local pode ser aumentada em 663,3 toneladas, sem exceder o limite de fósforo total recomendado na Resolução n 357/05 do CONAMA.

\section{REFERÊNCIAS}

ARANA, L.V. (2007) Aquicultura e desenvolvimento sustentável: subsídios para a formulação de políticas de desenvolvimento da aquicultura brasileira. Florianópolis: Editora da UFSC. 310 p.

APHA - AMERICAN PUBLIC HEALTH ASSOCIATION. (1998) Standard methods for the examination of water and wastewater. Washington: APHA. 824 p.
BOYD, C.E. \& TUCKER, C.S. (1998) Pond aquaculture water quality management. Boston: Kluwer Academic Publishers. 700 p.

BUENO, G.W:; MARENGONI, N.G.; GONÇALVES JÚNIOR, A.C.; BOSCOLO, W.R.; TEIXEIRA, R.A. (2008) Estado trófico e bioacumulação do fósforo total no cultivo de peixes em tanques-rede na área aquícola do reservatório de Itaipu. Acta Scientiarum Biological Sciences, v.30, n.3, p.237-243. 
CABRAL, J.B.P.; SANTOS, F.F.; NOGUEIRA, P.F.; BRAGA, C.C. (2012) Análise espacial de sólidos em suspensão em reservatórios do estado de Goiás: estudo de caso de UHE Caçu e Barra dos Coqueiros. Revista Geonorte, Edição Especial, v. 2, n. 4, p. 126-137.

CABI - COMITÊ DOS AFLUENTES DO BAIXO IGUAÇU. (2012) Descrição e diagnóstico da unidade hidrográfica dos afluentes do baixo Iguaçu com vistas à criação do comitê de bacia. 15 p. Disponível em: <http://www. recursoshidricos.pr.gov.br/arquivos/File/CERH_-_19_RO/caracterizacao_ diagnostico_ugrhi_baixo_iguacu.pdf>. Acesso em: 21 set. 2015.

CONAMA - CONSELHO NACIONAL DO MEIO AMBIENTE. (2005) Resolução no 357, de 17 de março de 2005. Dispõe sobre a classificação dos corpos de água e diretrizes ambientais para o seu enquadramento, bem como estabelece as condições e padrões de lançamento de efluentes, e dá outras providências. Brasília: Diário Oficial da União.

COSTA, B.D.F. (2004) Caracterização ambiental e dimensionamento da capacidade de aproveitamento do Reservatório de Sobradinho para a implantação de tanques-rede. Dissertação (Pós-graduação em Recursos Pesqueiros e Aquicultura) - Universidade Federal Rural de Pernambuco, Recife.

DILLON, P.J. \& RIGLER, F.H. (1974) A test of a simple nutrient budget model predicting the phosphorous concentration in a lake water. Journal of the Fisheries Research Board of Canada, v. 31, n. 11, p. 1771-1778.

DIEMER, O.; NEU, D.H.; FEIDEN, A.; LORENZ, E.K.; BITTENCOURT, F.; BOSCOLO, W.R. (2010) Dinâmica nictimeral e vertical das características limnológicas em ambiente de criação de peixes em tanques-rede. Ciência Animal Brasileira, v.1 1, n. 1, p. 24-31.

ECHANIZ, S. \& VIGNATTI, A. (2009) Determinación del estado trófico y de la capacidad de carga de embalse casa de piedra. BioScriba, v. 2, n. 1, p. 41-51.

ELER, M.N.; CAMPAGNA, A.F.; MINILLO, A.; RIBEIRO, M.A.P.; ESPINDOLA, E.L.G. (2009) Water quality, toxity and gill lesions caused by intraperitoneally administred water-bloom crude extract in Brycon Cephalus (Günter, 1986; Characidae) from fee-fishing ponds in São Paulo state, Brazil. Acta Limnologica Brasiliensia, v. 21, n. 1, p. 89-100.

ESTEVES, F.A. (1998) Fundamentos de limnologia. 2 ed. Rio de Janeiro: Interciência.

FREITAS, F.C.; SANTOS-SILVA, E.N.; DARWICH, A.J. (2011) Influência do cultivo de peixes em tanque- rede nos teores de nitrogênio e fósforo no lago Tupé, Manaus-AM. Revista BioTupé, n. 3, p. 53-59.
INFORMATIVO CULTIVANDO ÁGUA BOA. (2009) Caderno aquicultura III. Disponível em: <http://www.cultivandoaguaboa.com.br/sites/default/ files/iniciativa/Informativo_Cultivando_Agua_Boa_Especial_Educacao_ Ambiental.pdf>. Acesso em: 21 set. 2015.

IAP - INSTITUTO AMBIENTAL DO PARANÁ. (2012) Aspectos legais da Tilápia do Nilo em sistemas de cultivo em tanques redes nos reservatórios da bacia do Rio Iguaçu. Encontro, Boa Vista da Aparecida, Paraná.

KUBITZA, F. (1999) Qualidade da água na produção de peixes. Piracicaba: ESALQ.

KUBITZA F. (2000) Tilápia: tecnologia e planejamento na produção comercial. Jundiaí: Kubitza.

LIU, Y.; YANG, P.; HU, C.; GUO, H. (2008) Water quality modeling for load reduction under uncertainty: a Bayesian approach. Water Quality, v. 42, n.13, p. 3305-3314

PEREIRA, M.B. \& BLEICH, M.E. (2009) Variações limnológicas abióticas espaciais e temporais em uma lagoa na bacia Araguaia-Tocantins, Mato Grosso. Revista Iniciação Científica CESUMAR, v. 11, n. 1, p. 29-35.

PEREIRA, L.P.F. \& MERCANTE, C.T.J. (2005) A amônia nos sistemas de criação de peixes e seu efeitos sobre a qualidade da água: uma revisão. Boletim do Instituto de Pesca, v. 31, n. 1, p. 81-85.

SANTOS, I. (2001) Hidrometria aplicada. Curitiba: Instituto de Tecnologia para o Desenvolvimento. $372 \mathrm{p}$.

SIPAÚBA-TAVARES, L.H. (1995) Limnologia aplicada à aquicultura. Jaboticabal: FUNEP.

SCHMITTOU, H.R. (1993) High density fish culture in low volume cages. Singapura: American Soybean Association. 78 p.

TUNDISI, J.G. (2005) Gerenciamento integrado de bacias hidrográficas e reservatórios estudos de caso e perspectivas. In: NOGUEIRA, M.G.; HENRY, R.; JORCIN, A. Ecologia de reservatórios: impactos potenciais, ações de manejo e sistemas em cascata. São Carlos: Rima. p. 1-21.

WETZEL, R.G. (1993) Limnologia. Lisboa: Fundação Calouste Gulbenkian. 919 p.

ZAR, J.H. (2009) Biostatistical Analysis. 5 ed. New Jersey: Prentice-Hall. 